\title{
Lattice instabilities in hexagonal NiSi: A NiAs prototype structure
}

\author{
Damien Connétable ${ }^{1, *}$ and Olivier Thomas ${ }^{2,3}$ \\ ${ }^{1}$ CIRIMAT (UMR 5085), ENSIACET, CNRS-INPT-UPS, 4 allée Emile Monso, BP 44362, F-31432 Toulouse Cedex 4, France \\ ${ }^{2}$ IM2NP, Aix-Marseille Université \\ ${ }^{3}$ IM2NP (UMR 6242), Faculté des Sciences et Techniques, CNRS, Campus de St. Jérome, F-13397 Marseille Cedex, France \\ (Received 19 January 2009; revised manuscript received 13 July 2009; published 23 February 2010)
}

\begin{abstract}
We report a first-principles study of the hexagonal NiSi phase with the $\mathrm{B} 8_{1}$ strukturbericht designation. This structure, reported by Föll [Philos. Mag. A 45, 31 (1982)], d'Heurle [J. Appl. Phys. 55, 4208 (1984)], and Dai [Appl. Phys. Lett. 75, 2214 (1999)], is actually only observed during annealing of Ni films on (111) silicon crystals. We discuss, in this paper, about its structural, energetic, vibrational, electronic, and elastic properties, computed by means of the density-functional and density-functional perturbative theory within the spinpolarized Perdew-Burke-Ernzerhof functional. Two configurations with this crystallographic structure have been studied, noted $h$-NiSi and $h$-SiNi in the following. We show that theoretical and experimental lattice parameters are not compatible for both systems. A large discrepancy $(8-10 \%)$ is evidenced, much larger than both experimental and simulation accuracies obtained for others Ni-Si systems. Moreover the vibrational spectra of $h$-NiSi and $h$-SiNi present both soft modes, indicating that in their ground states these systems are dynamically unstable. Using a band folding approach, we have analyzed modes for $h$-NiSi on a supercell, permitting us to identify eigenvectors associated to these instabilities. We have then relaxed the cell in accordance to these eigenvectors, and a final structure is thus proposed. To understand the mechanism at the origin of these negative frequencies in $h$-NiSi, electronic states around the Fermi level have been plotted, and we identify in the Fermi-surface potential nesting vectors, suggesting that an electron-phonon coupling mechanism could be at the origin of the instability. Whereas the ground state of " $h-\mathrm{NiSi}$ " seems not to be associated to the $\mathrm{B} 8{ }_{1}$ system, we show that a stress in the basal plane could induce an increasein the $\langle c\rangle$ axis, restoring the agreement with experimental data.
\end{abstract}

DOI: 10.1103/PhysRevB.81.075213

PACS number(s): 71.20.-b, 63.20.-e, 64.60.My

\section{INTRODUCTION}

$\mathrm{NiSi}[$ or $\mathrm{Ni}(\mathrm{Pt}) \mathrm{Si}$ ] is a low-resistivity intermetallic, which has attracted considerable attention as a contact material for advanced MOS devices. NiSi crystallizes in the orthorhombic $\mathrm{MnP}$ structure but, under specific experimental conditions, Föll, ${ }^{1}$ d'Heurle, ${ }^{2}$ and recently $\mathrm{Dai}^{3}$ have identified a hexagonal NiSi structure. This structure, not present in the common phase diagram of $\mathrm{NiSi}^{4}{ }^{4}$ has been reported during the growth of nickel silicide films on (111) silicon. From their experimental results, they have deduced that this "new" hexagonal phase seems to have the same crystallographic structure as NiAs prototype (B8 1 strukturbericht designation, No 194), a hexagonal structure with two nickel and two silicon atoms per unit cell (see Fig. 1). The lattice parameters have been deduced from transmission electron microscopy by d'Heurle ${ }^{2}$ and Dai: ${ }^{3} a_{h}=3.28 \AA$ and $c_{h}=5.23 \AA$, respectively. Atomic positions are, however, not known.

This structure has only been observed in thin films on (111) $\mathrm{Si}$. Otherwise the main observed NiSi structure is the orthorhombic $\mathrm{MnP}$ structure (noted $o-\mathrm{NiSi}$ ). To explain the presence of hexagonal phase, authors ${ }^{1,2}$ argue that $o-\mathrm{NiSi}$ phase can be described as a distorted hexagonal structure. Hexagonal lattices can be viewed as sublattices of orthorhombic unit cells and reciprocally a true hexagonal system can be obtained if the ratio $c_{o} \sqrt{3} / b_{o}$ is equal to 1 . For $o-\mathrm{NiSi}$, this ratio is experimentally very close to 1 (exactly 1.003). Since the experimental assignment of hexagonal NiSi structure is rather difficult its very existence is still debated and the question of the stability of this phase remains an un- solved question. This hexagonal phase is not described in the literature and its stabilization by stresses remains open.

The aim of this paper is to investigate the stability and the properties of this phase from first-principles calculations. In the NiAs prototype, the environment of the $\mathrm{Ni}$ atoms is fcclike and the environment of the As atoms is hop-like. One deduces two possibilities to arrange nickel and silicon atoms in the cell, the two Wyckoff sites $(2 a$ and $2 c)$ are consequently not equivalent. We have studied in this paper the two NiAs configurations noted $h$-NiSi and $h$-SiNi. We provide thus a description of structural, energetic, electronic, and vi-

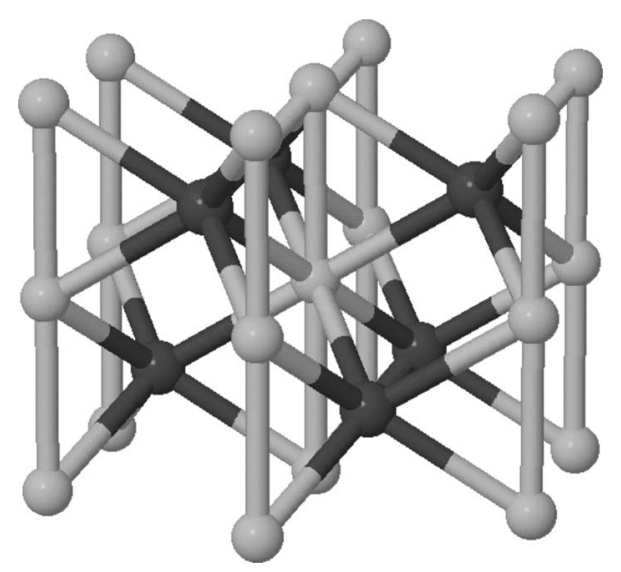

FIG. 1. Schematic representation of the hexagonal NiAs unit cell: nickel and arsenic atoms are, respectively, represented in green and in black. 
TABLE I. Optimized and experimental lattice parameters (in $\AA$ ), formation energies $E_{o}$ (in meV/atom), and total magnetic moments (in Bohr's magneton $\mu_{B}$ ) of the Ni-Si alloys. For the intermediate and final structures of NiSi, the structure is monoclinic, we report $\alpha, \beta$, and $\gamma$.

\begin{tabular}{|c|c|c|c|c|c|c|c|c|}
\hline Phase & & Pearson symbol & Strukturbericht Designation & $\begin{array}{l}a_{o} \\
(\AA)\end{array}$ & $\begin{array}{c}b_{o} \\
(\AA)\end{array}$ & $\begin{array}{c}c_{o} \\
(\AA)\end{array}$ & $\begin{array}{c}E_{o} \\
\text { (meV/atom) }\end{array}$ & $\mu_{B}$ \\
\hline NiSi-NiAs & Expt. $^{\mathrm{a}}$ & hP4 (194) & $\mathrm{B} 8_{1}$ & 3.28 & & 5.23 & & \\
\hline \multirow[t]{3}{*}{$h$-NiSi } & PAW & & & 3.50 & & 4.82 & -404 & 0.00 \\
\hline & US & & & 3.51 & & 4.83 & -395 & 0.00 \\
\hline & FLAPW & & & 3.50 & & 4.83 & & \\
\hline \multirow[t]{2}{*}{$h$-SiNi } & PAW & & & 3.54 & & 4.87 & -148 & 0.00 \\
\hline & US & & & 3.53 & & 4.87 & -146 & 0.00 \\
\hline NiSi disordered & PAW & & & 3.68 & & 4.63 & -138 & 0.00 \\
\hline NiSi intermediate & US & $\alpha=\beta=90^{\circ}$ & $\gamma=58^{\circ}$ & 6.92 & 5.55 & 5.14 & -460 & 0.00 \\
\hline NiSi final & US & $\alpha=\beta=90^{\circ}$ & $\gamma=61^{\circ}$ & 6.55 & 6.55 & 5.16 & -495 & 0.00 \\
\hline NiSi stressed & US & hP4 (194) & $\mathrm{B} 8_{1}$ & 3.28 & & 5.29 & -325 & 0.00 \\
\hline \multirow[t]{2}{*}{$o-\mathrm{NiSi}$} & Expt. $^{b}$ & oP8 (62) & B31 & 5.18 & 3.33 & 5.62 & $-447^{\mathrm{c}}$ & \\
\hline & PAW $^{\mathrm{d}}$ & & & 5.17 & 3.38 & 5.62 & -503 & 0.00 \\
\hline
\end{tabular}

aReference 3 .

${ }^{\mathrm{b}}$ Reference 12 .

${ }^{\mathrm{c}}$ Reference 13 .

${ }^{\mathrm{d}}$ Reference 14

brational properties from first-principles calculations of these two systems.

\section{COMPUTATIONAL DETAILS}

The ground states have been performed using the computational implementation of the density-functional theory (DFT) Vienna $a b$ initio simulation package [VASP (Ref. 5)]. Projected augmented waves (PAW) pseudopotential approach $^{6}$ and the Perdew-Burke-Ernzerhof ${ }^{7}$ (PBE) generalized gradient approximation of the exchange and correlation functional within its spin-polarized version have been used. Lattice parameters have been optimized thanks to the conjugate gradient method. We have employed a $400 \mathrm{eV}$ energy cutoff and a dense mesh grid $(10 \times 10 \times 10)$ to optimize ground state. Electronic density of states and formation energy have been calculated on a finest grid $(15 \times 15 \times 15)$ and a higher energy cutoff $(500 \mathrm{eV})$.

We have employed an other DFT package [QUANTUMESPRESSO (Ref. 8)] with an other type of pseudopotential approach (ultrasoft pseudopotentials 9 according to a modified Rappe-Rabe-Kaxiras-Joannopoulos scheme following the method of Ref. 10) to corroborate and to complete VASP simulations. NiSi phases have been optimized thanks to the first-principles implementation of a variable cell-shape damped molecular dynamics (VCSDMD). ${ }^{11}$

\section{RESULTS AND DISCUSSION}

\section{A. Stability of NiSi structures}

In Table I, we have reported optimized lattice parameters as well as magnetic moment $\mu_{o}$ per unit cell of $h$-NiSi,
$h$-SiNi, and $o$-NiSi for comparison. Experimental data are reported too.

Whereas calculated and experimental $o$-NiSi lattice parameters are compatible, optimized $h$-NiSi and $h$-SiNi lattice parameters are significantly different from experimental values. These decrepancies are much larger than simulation accuracy: around $8-10 \%$ to be compared to $1-2 \%$ for $o$-NiSi. One notes that $a_{h}^{\text {theo }}$ is greater than $a_{h}^{\exp }$ while $c_{h}^{\text {theo }}$ is smaller than $c_{h}^{\exp }$. Distances between nickel atoms in $h$-NiSi are theoretically slightly smaller in the reference state than experimental ones $[d(\mathrm{Ni}-\mathrm{Ni}, \mathrm{NiSi}-$ theo $)=2.41 \AA, d(\mathrm{Ni}-\mathrm{Ni}$, $\mathrm{Ni}-\mathrm{fcc})=2.48 \AA$, and $d(\mathrm{Ni}, \mathrm{Ni}, \mathrm{NiAs}-\mathrm{exp})=2.66 \AA]$. A contrario, distances between silicon atoms and silicon and nickel atoms are larger in theory as compared with experiment $[d(\mathrm{Ni}-\mathrm{Si})=2.36$ and $2.30 \AA$, theoretically and experimentally, respectively].

Formation energies (computed with ferromagnetic fcc-Ni and diamond silicon phases as reference states) are found negative: around -400 and $-145 \mathrm{meV} /$ atom for $h$-NiSi and $h$-SiNi, respectively. These phases are less stable than $o$-NiSi $[\simeq-500 \mathrm{meV} /$ atom (Ref. 14)], which suggests that hexagonal NiSi structures could be metastable phases. Nevertheless, the strong differences between experimental and theoretical lattice parameters cannot be explained. We have performed lattice parameters and formation energies calculations with the ESPRESSO package. The values $\left(a_{h}=3.51 \AA\right.$ and $c_{h}$ $=4.83 \AA,-395 \mathrm{meV} /$ atom for $h-\mathrm{NiSi}$, and $a_{h}=3.53 \AA$ and $c_{h}=4.87 \AA,-146 \mathrm{meV} /$ atom for $h$-SiNi) are found in agreement with PAW simulations. ${ }^{15}$

It is important to point out that we have checked that these optimized structures do not correspond to a local minimum and do not depend on the choice of the functional and the pseudopotentials. Indeed we have tried others functionals commonly used: local-density approximation (LDA) (Ref. 

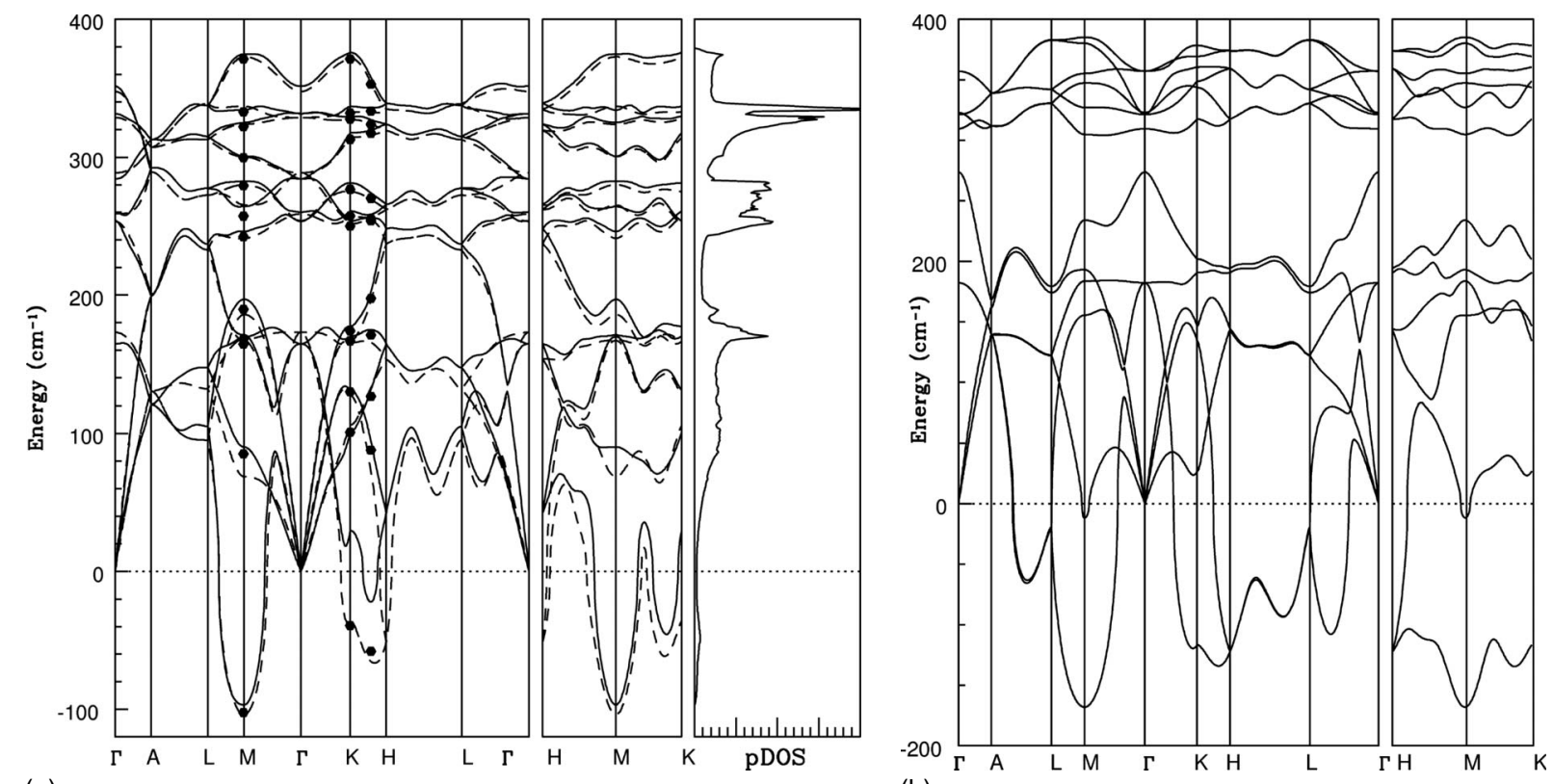

(a)

(b)

FIG. 2. Vibrational band structure and density of states of $h$-NiSi at the optimized lattice parameters (left) and at the experimental lattice parameters (right). The imaginary values of frequencies are plotted along negative frequency axes. The dashed lines represent a $(3,3,3)$ grid simulation, and lines $(6,6,6) \mathbf{q}$ mesh grid (see text). Dots are the frequencies calculated directly with high convergence parameters: in $\mathbf{M}$ and $\mathbf{K}$ and a point between $\mathbf{K}-\mathbf{H}$ direction.

16) and generalized gradient approximation (GGA). ${ }^{17}$ Lattice parameters are found very close to previous $\mathrm{PBE}$ theoretical results: $a=3.45 \AA$ and $c=4.83 \AA$, and $a=3.52 \AA$ and $c$ $=4.89 \AA$, for LDA and GGA simulations, respectively. Moreover, full-potential linearized augmented plane-wave (FLAPW) simulations have been used to confirm our results (see Table I).

One finds that NiSi structures are metallic with low densities of state at the Fermi level (see Fig. 4), and without ferromagnetic behavior. From a strict energetic point of view, $h$-NiSi seems to be a better candidate for a comparison with experiments than $h$-SiNi.

For sake of completeness we have also investigated the intermediate structure where one nickel atom is interchanged with one silicon atom. The lattice parameters are sensibly the same than $h$-NiSi or $h$-SiNi. This theoretical simulation, noted NiSi disordered in the Table I, is not able to solve the disagreement.

\section{B. Phonon dispersion relations}

In order to determine the full phonon dispersions of hexagonal NiSi phases, the interatomic force constants $[\mathrm{C}(\mathbf{R})]$ have been computed by Fourier transformation of the dynamical matrices calculated on a given $\mathbf{q}$ points grid. The phonon dispersion, along high symmetry directions, of the Brillouin zone has been then obtained by interpolating the dynamical matrices calculated on a finest grid from $\mathrm{C}(\mathbf{R})$ calculated previously. The vibrational density of states have been evaluated on a $30 \times 30 \times 30$ tetrahedron $\mathbf{q}$ mesh.

\section{1. $h$-NiSi}

We have studied the influence of the cut-off energy and $\mathbf{k}$ mesh grid on the accuracy of the vibrational properties for $h$-NiSi. The dynamical matrix has been computed on a 6 $\times 6 \times 6$ q mesh grid with 20 Ry energy, and 200 Ry for the charge-density cutoff and wave-function cutoff, respectively, and a $10 \times 10 \times 10 \mathbf{k}$ mesh grid. The result is plotted in Fig. 2 (dashed lines). An increase in the cut-off energies (40 Ry and $400 \mathrm{Ry})$ and $\mathbf{k}$ mesh grid $(15 \times 15 \times 15)$, but smaller $\mathbf{q}$ grid $(3 \times 3 \times 3)$ does not modify significantly phonon spectra (thick lines Fig. 2). The main differences are along the K-H direction, where new soft modes appear. It is then essential, for the following, to identify numerical artifacts.

The great interest of the DFPT is to compute dynamical frequencies at any $\mathbf{q}$ point in the $\mathrm{Bz}$ (monochromatic pertur-

TABLE II. Influence of the smearing broadening on frequencies at the zone boundary point $\mathbf{M}$ in the $h$-NiSi system.

\begin{tabular}{|c|c|c|c|c|c|c|c|c|c|c|c|c|}
\hline \multirow{2}{*}{$\begin{array}{c}\begin{array}{c}\sigma \\
(\mathrm{Ry})\end{array} \\
0.03\end{array}$} & \multicolumn{12}{|c|}{$\begin{array}{l}\text { Frequencies } \\
\quad\left(\mathrm{cm}^{-1}\right)\end{array}$} \\
\hline & -113 & 67 & 157 & 168 & 182 & 246 & 257 & 278 & 299 & 320 & 333 & 372 \\
\hline 0.01 & -102 & 88 & 166 & 169 & 190 & 242 & 259 & 279 & 300 & 323 & 333 & 371 \\
\hline 0.005 & -102 & 85 & 164 & 168 & 190 & 242 & 257 & 279 & 299 & 322 & 333 & 371 \\
\hline
\end{tabular}




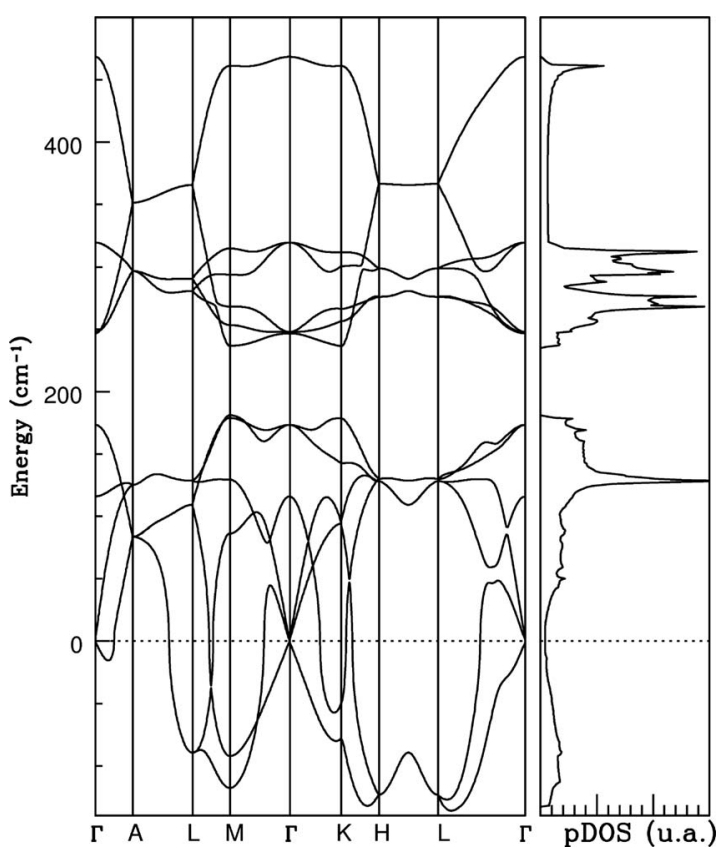

FIG. 3. Vibrational band structure and density of states of $h$-SiNi.

bation) with the same unit cell. The frequencies thus calculated must correspond with interpolated frequencies obtained from $\mathrm{C}(\mathbf{R})$ at the same $\mathbf{q}$ point. We have then directly calculated the frequencies, for different well chosen $\mathbf{q}$ points, to verify the accuracy of interpolated frequencies: at $\mathbf{K}$ and $\mathbf{M}$, and at the negative frequency between $\mathbf{K}-\mathbf{H}$. We have adopted high convergence criteria (50 Ry, $500 \mathrm{Ry}$, and 15 $\times 15 \times 15 \mathbf{k}$ grid) for these calculations. Results have been added in Fig. 2 (dots). In $\mathbf{M}$, we have also evaluated the effect of the smearing parameter on frequencies $(0.03,0.01$, and 0.005 Ry) see Table II. One deduces from these simula- tions that, on one hand, the effect of the smearing is negligible, which could suggest that temperature effect should be weak, and on the other hand, that direct calculations are well correlated with the interpolated points on a $(3,3,3) \mathbf{q}$ grid (used in the following). ${ }^{18}$ These results indicate that the best description of the vibrational properties is obtained with the finest description of the Fermi surface, but not necessarily with a fine $\mathbf{q}$ mesh grid. One conclude that there is more than one soft mode in the phonon dispersion curves of $h$-NiSi.

The most striking features of the calculated vibrational properties of $h$-NiSi are the instabilities which appear over a large area in reciprocal space. There are negative/imaginary vibrational modes along different directions: in $\mathbf{M}$ and along $\mathbf{M}-\mathbf{K}$ and $\mathbf{K}-\mathbf{H}$ directions. These results suggest that the stoichiometric compound is dynamically unstable in the harmonic approximation.

We have verified that the dynamical properties of $h-\mathrm{NiSi}$ using the experimental lattice parameters (see Fig. 2) do not change our conclusion. A new soft mode at the $\mathbf{L}$ point is appearing by imposing experimental lattice parameters. Moreover, vibrational properties of the $o$-NiSi (see Ref. 14) does not present similar negative modes. These results suggest also that, in its theoretical ground-state configuration, $h$-NiSi should induce a phase transition.

To complete the understanding of the dynamical properties of $h$-NiSi, we have investigated the eigenvectors associated to these imaginary modes, to check the stable phase. Since the instabilities are associated with soft modes at the Brillouin zone boundaries, we have employed a band folding approach to calculate them. Frequencies on a $2 \times 2 \times 1$ $h$-NiSi supercell by means of the density-functional perturbative theory in $\boldsymbol{\Gamma}$ have been computed. Three negative frequencies, which are corresponding to the imaginary frequencies in $\mathbf{M}$ and $\mathbf{K}$ (band folding), have been found. In Fig. 6, we give a schematic representation of these three eigenvectors. One notices that they are mainly localized on silicon
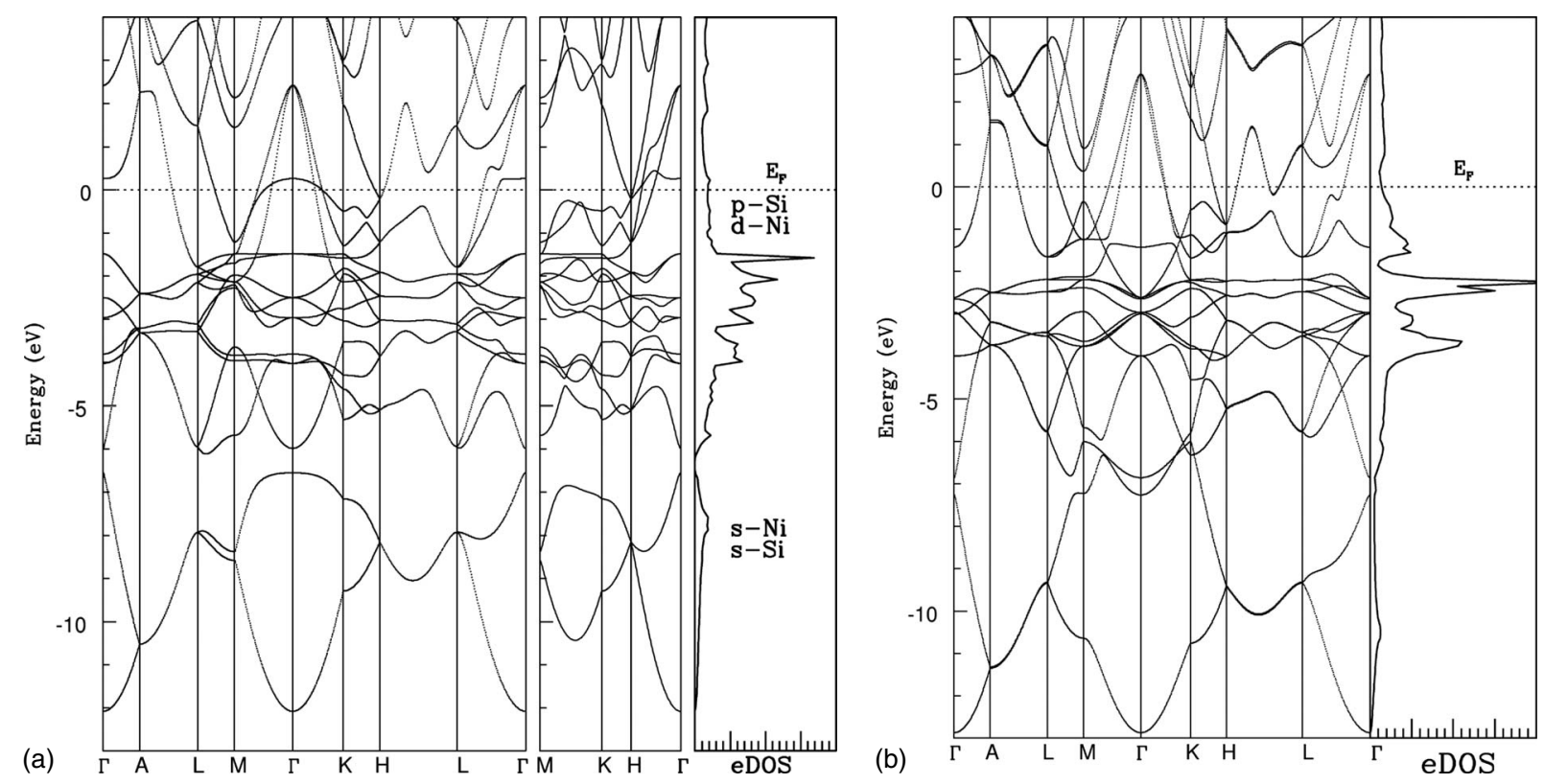

FIG. 4. Electronic band structure and density of states of $h$-NiSi (left) and $h$-SiNi (right). 

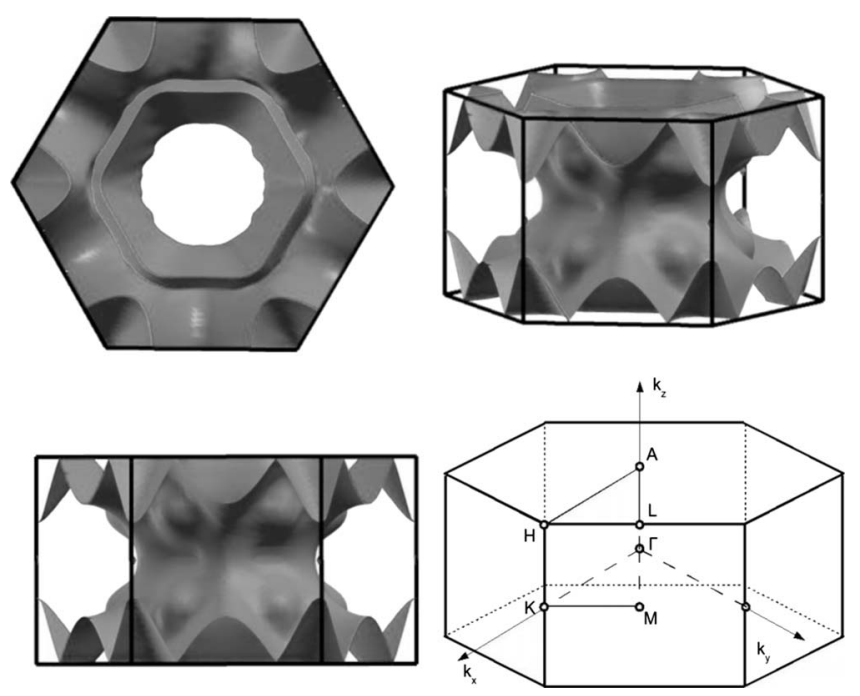

FIG. 5. Three different views of the Fermi surface of $h$-NiSi have been drawn. The fourth image represents the Brillouin zone.

atoms. From these eigenvectors, we have been looking for the " $h$-NiSi" relaxed structure - on a $2 \times 2 \times 1$ supercell-by means of the VCSDMD. Each three deformations applied have yielded the same structure: a monoclinic structure (see Table I at the label "NiSi intermediate"). However, a study of vibrational modes in zone center reveals that this structure has one soft mode too and is then not stable. We have relaxed again this intermediate structure in accordance with these last eigenvectors of the NiSi-intermediate phase. The final structure, which does not have negative frequencies, is a distorted hexagonal phase (monoclinic). A schematic representation of the intermediate and final phases is given in Fig. 7 (labeled NiSi final).

\section{2. $h-\mathrm{SiNi}$}

Using the same criteria of convergence as the previous one, vibrational properties of $h$-SiNi have been calculated. Results on this system are similar to those obtained for $h$-NiSi (see Fig. 3). One notes lots of negative modes along different crystallographic directions $(\mathbf{M}, \mathbf{K}, \mathbf{H}, \mathbf{L})$. In addition, negative frequencies are appearing around $q=\Gamma$, indicating that this system is furthermore mechanically unstable. In the following, we focus on $h$-NiSi.
TABLE III. Elastic constants (in GPa) of $h$-NiSi $\left[C_{66}=\left(C_{11}\right.\right.$ $\left.\left.-C_{12}\right) / 2\right]$.

\begin{tabular}{lccccccc}
\hline \hline & $C_{11}$ & $C_{12}$ & $C_{13}$ & $C_{33}$ & $C_{44}$ & $C_{66}$ & $B_{v}$ \\
\hline$h$-NiSi & 231 & 151 & 106 & 317 & 121 & 40 & 167 \\
\hline \hline
\end{tabular}

\section{Elastic properties}

We have computed elastic constants $C_{i j}$ (namely, $C_{11}, C_{12}$, $C_{33}, C_{13}$, and $\left.C_{44}\right)$ of $h$-NiSi taking into account atomic relaxations. We have reported them in the Table III. Criteria of stability (see Ref. 19) are all verified for this system, i.e., (i) all $C_{i j}$ are positive, (ii) $C_{11}-C_{12}>0$, (iii) $C_{11}+C_{33}+C_{12}>0$, and (iv) $\left[\left(C_{11}-C_{12}\right) C_{33}-2 C_{13}^{2}\right]>0$. From a strict mechanical point of view $h$-NiSi is stable in agreement with calculated phonon dispersion curves.

\section{Electronic properties}

For the sake of completeness, we have plotted the electronic band structure along high-symmetry points of the first Brillouin zone and density of states as shown Fig. 4, with the energy zero corresponding to the valence-band top. As we have noted previously, $h$-NiSi and $h$-SiNi are metallic systems without ferromagnetic behavior. The Fermi state consists of silicon $p$ states and nickel $d$ states, in accordance with other Ni-Si alloys. ${ }^{14}$ The density of states at the Fermi level are found low.

For further informations, we have drawn different representations of the Fermi surface of $h$-NiSi in Fig. 5. Along $\langle c\rangle$ axis ( $\boldsymbol{\Gamma}$-L direction), one can identify a depletion of electronic states. In the basal plane ( $\boldsymbol{\Gamma}-\mathbf{M}-\mathbf{K}$ directions), the Fermi surface exhibits nesting features, which may explain the soft modes that appear in the phonon dispersion curves. Indeed, bands cross the Fermi level in between $\boldsymbol{\Gamma}$-M and $\boldsymbol{\Gamma}-\mathbf{K}$ directions. Concerning the soft mode along $\mathbf{M}-\mathbf{K}$ directions, one can associate it to a nesting of the Fermi surface along the $\mathbf{L - H}$ directions of the Brillouin zone.

\section{E. Discussion}

Although systems which exhibit lattice instabilities have been already reported in the literature (see, for example, Refs. 20 and 21), the experimental lattice parameters do agree with the calculated ones. In our case the large discrep-

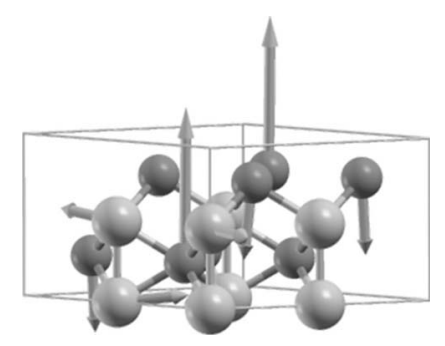

(a)

(b)

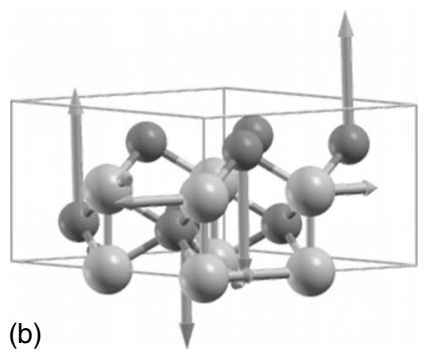

(c)

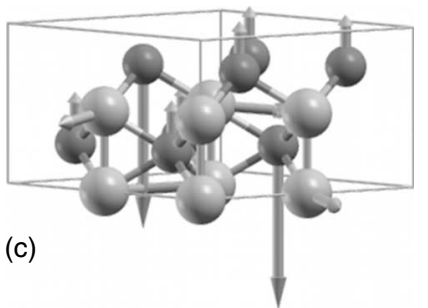

FIG. 6. Schematic representation of the eigenvectors of the soft modes for $h$-NiSi. Silicon atoms are in dark and nickel atoms in gray. 

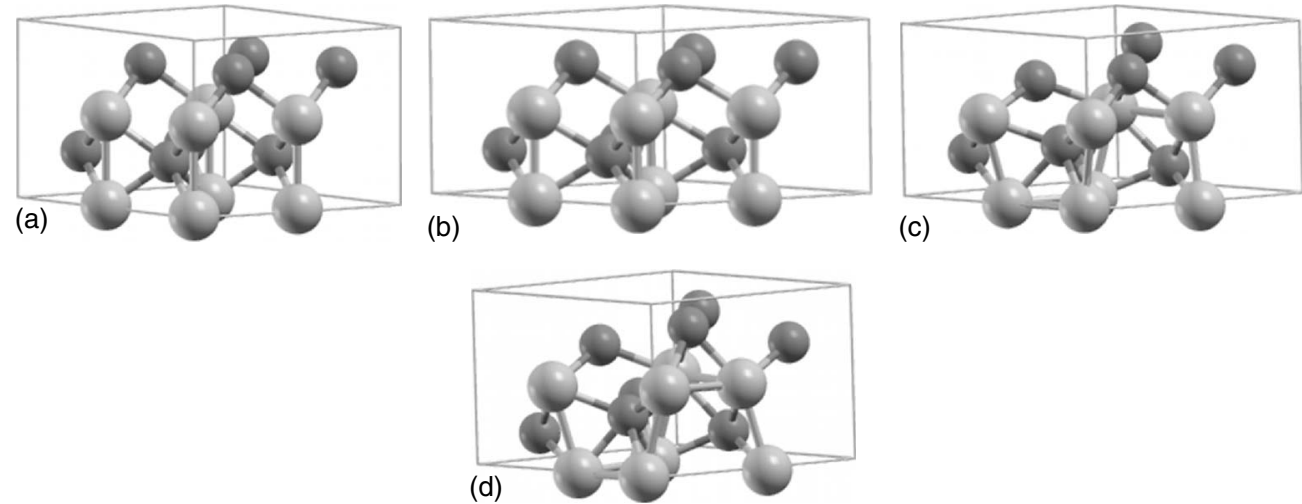

FIG. 7. Schematic representation of the $2 \times 2 \times 1$ supercell, from left to right: stressed system in the basal plane, initial (optimized $h$-NiSi system), intermediate, and final structures.

ancy for $h$-NiSi and $h$-SiNi between experimentally reported lattice parameters and calculated ones raises questions.

One may argue that some of the experimentally reported results ${ }^{3}$ concern $\mathrm{Pt}$-alloyed $\mathrm{NiSi}$ films. The presence of $\mathrm{Pt}$ might be invoked to explain an eventual stabilization of the hexagonal phase. However, earlier reports of hexagonal NiSi referred to Pt-free films.

Stabilization of hexagonal $\mathrm{NiSi}$ by epitaxial stresses is another possible explanation that can be put forward. To verify this hypothesis, we have simulated a stressed structure on a $2 \times 2 \times 1$ supercell $h$-NiSi. We have held up relaxation in the hexagonal plane by imposing the experimental value of the lattice parameter $a_{o}$, and we have relaxed structure along the $\langle c\rangle$ axis to start with $c_{o}=4.82 \AA$. This approach should simulate an epitaxial NiSi phase on a sixfold symmetry surface of the underlying (111) plane of the Si substrate. In this configuration, the in-plane lattice parameter $a_{o}$ is imposed by the substrate. This simulation (noted NiSi stressed in the Table I) yields a correct NiSi-NiAs structure with a lattice parameter $c_{o}$ close to the experimental value. In this configuration, the structure does not present imaginary frequencies. In the basal plane, a residual stress remains (around $11 \mathrm{GPa}$ ). If the system is fully relaxed, the $h-\mathrm{NiSi}$ lattice parameters are recovered. This numerical experiment suggests that the elastic distortion induced by epitaxy may explain the discrepancy between calculated and experimental lattice parameters.

\section{CONCLUSION}

This work has been motivated by the experimental finding of hexagonal NiSi with NiAs structure in thin films grown on $\mathrm{Si}$. This NiSi hexagonal structure is not reported as a stable phase in the equilibrium diagram and we therefore decided to perform a first-principles study of hexagonal NiSi with NiAs structure. We have shown that the two potential phases are thermodynamically stable with a negative formation energy. Furthermore both phases are metallic and are not ferromagnetic. The investigated hexagonal NiSi structures are, however, dynamically unstable as indicated by negative vibrational energies. Compressing this structure in the hexagonal plane yields a $c$-lattice parameter in good agreement with experiments. The predicted dynamical unstability indicates, however, that the experimentally observed NiSi structure is not hexagonal NiSi with NiAs type.

\section{ACKNOWLEDGMENTS}

The authors acknowledge use of the supercomputer facilities at Calmip CICT Toulouse, France. We thank G. Hug (ONERA) for FLAPW calculations.

\footnotetext{
*damien.connetable@ensiacet.fr

${ }^{1}$ H. Föll, P. S. Ho, and K. N. Tu, Philos. Mag. A 45, 31 (1982).

${ }^{2}$ F. d'Heurle, C. S. Petersson, J. E. E. Baglin, S. J. La Placa, and C. Y. Wong, J. Appl. Phys. 55, 4208 (1984).

${ }^{3}$ J. Y. Dai, D. Mangelinck, and S. K. Lahiri, Appl. Phys. Lett. 75, 2214 (1999).

${ }^{4}$ T. B. Massalski, P. R. Subramanian, H. Okamoto, and L. Kacprzak, Binary Alloy Phase Diagrams, 2nd ed. (ASM International, Materials Park, OH, 1990).

${ }^{5}$ G. Kresse and J. Hafner, Phys. Rev. B 47, 558 (1993); 49, 14251 (1994); G. Kresse and J. Furthmüller, ibid. 54, 11169 (1996); Comput. Mater. Sci. 6, 15 (1996).

${ }^{6}$ G. Kresse and D. Joubert, Phys. Rev. B 59, 1758 (1999).
}

${ }^{7}$ J. P. Perdew, K. Burke, and M. Ernzerhof, Phys. Rev. Lett. 77, 3865 (1996); 78, 1396 (1997).

${ }^{8}$ quantum-ESPRESSO is a community project for high-quality quantum-simulation software, based on density-functional theory, and coordinated by Paolo Giannozzi. See http:// www.quantum-espresso.org and http://www.pwscf.org

${ }^{9}$ D. Vanderbilt, Phys. Rev. B 41, 7892 (1990).

${ }^{10}$ A. M. Rappe, K. M. Rabe, E. Kaxiras, and J. D. Joannopoulos, Phys. Rev. B 41, 1227 (1990).

${ }^{11}$ R. M. Wentzcovitch, J. L. Martins, and G. D. Price, Phys. Rev. Lett. 70, 3947 (1993).

${ }^{12}$ D. F. Wilson and O. B. Cavin, Scr. Metall. Mater. 26, 85 (1992).

${ }^{13}$ W. Oelson and H. Samson-Himmelstjerna, Mitt. Kaiser-Wilhelm 
Inst. Eisenforch. Düsseldorf 18, 131 (1936).

${ }^{14}$ D. Connétable and O. Thomas, Phys. Rev. B 79, 094101 (2009).

${ }^{15}$ The convergence of lattice parameters and formation energies have been verified through an increase the cut-off energy and $\mathbf{k}$ mesh grid $[600 \mathrm{eV}$ and a $(30,30,30) \mathbf{k}$ grid] without significant modification.

${ }^{16}$ J. P. Perdew and A. Zunger, Phys. Rev. B 23, 5048 (1981).

${ }^{17}$ Y. Wang and J. P. Perdew, Phys. Rev. B 44, 13298 (1991).

${ }^{18}$ The effect of the cut-off energies and the $\mathbf{k}$ mesh grids on frequencies have been analyzed carefully. Three additional intermediates criteria of convergence have been studied: two simulations with the same $\mathbf{q}$ grid and cut-off energy $(3 \times 3 \times 3$ and 20
Ry) with $15 \times 15 \times 15$ and $10 \times 10 \times 10 \mathbf{k}$ grids, and a third with a finest $\mathbf{q}$ grid and a high cut-off energy. $(6 \times 6 \times 6$ and $40 \mathrm{Ry})$ and a $10 \times 10 \times 10 \mathbf{k}$ grid. The better precision has been obtained with the better description of the electronic band structure (40 Ry and the finest $\mathbf{k}$ mesh) and a suitable description of the interatomic force constants $(3 \times 3 \times 3 \mathbf{q}$ grid $)$.

${ }^{19}$ R. A. Cowley, Phys. Rev. B 13, 4877 (1976).

${ }^{20}$ X. Huang, C. Bungaro, V. Godlevsky, and K. M. Rabe, Phys. Rev. B 65, 014108 (2001).

${ }^{21}$ C. Bungaro, K. M. Rabe, and A. Dal Corso, Phys. Rev. B 68, 134104 (2003). 